\title{
Comments on: "Phrenic Nerve Block, the Rescue Management for Pneumothorax after Retrolaminar Block"
}

\author{
Kartik Sonawane $^{1}$, Elayavendhan Kuppusamy ${ }^{2}$, Tuhin Mistry ${ }^{3 *}$, Joseph Abraham \\ Poonuraparampil ${ }^{4}$ \\ ${ }^{1,2,3}$ Department of Anaesthesiology, Ganga Medical Centre \& Hospitals Pvt Ltd, Coimbatore, India \\ ${ }^{4}$ Department of Anaesthesiology, All India Institute of Medical Sciences, Raipur, India
}

\section{Dear Editor,}

We read with interest the recent publication by Mishra et al. in the June 2020 issue of Sri Lankan Journal of Anaesthesiology. ${ }^{1}$ We appreciate the authors for sharing this interesting case. However, we have the following concerns:

1. Intraoperative development of pneumothorax during robotic laparoscopic nephrectomy could result from single or multiple causes, as mentioned by authors. In the reported scenario, spontaneous rupture of pulmonary bulla probably caused the pneumothorax. Iatrogenic puncture of pleura during Retrolaminar block (RLB) is a rare possibility, as the pleura lies more anterior and lateral to the site of injection.

2. Pfeiffer et al. described the anatomical landmark guided RLB as a simple alternative to traditional paravertebral block to minimize the chances of pleural injury. Later, mandatory use of ultrasonography (USG) has been suggested to correctly identify the plane between thoracic laminae and deep paraspinal muscles and minimize the risk of epidural injection landmark technique. ${ }^{2}$ So, it

*Correspondence: Tuhin Mistry

E mail: tm.tuhin87@gmail.com

https://orcid.org/0000-0003-1904-4831

Received: $12 / 12 / 2020$

Accepted: 08/09/2021

DOI: https://doi.org/10.4038/slja.v29i2.8735 is prudent to use real-time USG guidance during RLB to avoid any inadvertent complications.

3. Pneumothorax is a recognized complication of laparoscopic surgery, with an incidence of $0.01-0.4 \% .^{3}$ According to the authors, pneumothorax was diagnosed after extubation and resolved in 24 hours. Intraoperative chest auscultation or ultrasound scanning could have helped in the early diagnosis of pneumothorax in a patient of chronic obstructive pulmonary disease (COPD) with a rise in end-tidal $\mathrm{CO}_{2}$ and airway pressures. Moreover, the stable intraoperative haemodynamics and other clinical parameters also indicate the possibility of capnothorax, which can resolve spontaneously without invasive management. $^{3}$

4. Phrenic nerve block (PNB) causes an ipsilateral hemi-diaphragmatic palsy (HDP), resulting in loss of cephalocaudal movements of the diaphragm during respiration. Consequently, it will reduce the patient's ability to generate negative pressure to expand the lung. Hence, PNB, especially in a COPD patient, may not be an ideal choice, for the management of pneumothorax.

5. The authors used an initial bolus of $0.5 \%$ bupivacaine $20 \mathrm{ml}$, followed by two doses of $0.25 \%$ bupivacaine $15 \mathrm{ml}$ at 6 hours and 12 hours for USG guided PNB. Phrenic nerve lies very close to the brachial plexus (within $2 \mathrm{~mm}$ ) at the level of cricoid cartilage or sixth cervical vertebra. ${ }^{4}$ Hence, administration of such large volumes of local anaesthetic may result in inadvertent brachial plexus block 
and/or other complications. Moreover, the identification of phrenic nerve may not always be feasible, even with the highresolution $\mathrm{USG}^{4}$

6. Rupture of the pleural bleb can cause primary spontaneous unstable pneumothorax, which may progress to tension pneumothorax, requiring immediate drainage. HDP following PNB results in cephalad shifting of the ipsilateral diaphragm. Thus, reducing the volume of the chest cavity. The air leak from open bulla may further increase pressure inside the chest, which may worsen the clinical course of pneumothorax.

7. In primary pneumothorax, the air is collected in intra-pleural (between two pleural layers) and extra-pleural (inside the chest cavity) spaces. The collected air may cause pleuritic pain leading to laboured breathing. PNB in this scenario helps to reduce this pain making breathing more comfortable. Therapeutic use of PNB in other types of pneumothorax may not be helpful, instead, it may worsen symptoms.

\section{References}

1. Mishra P, Talwar P, Govil $\mathrm{N}$ and Parameswaran P. Phrenic nerve block, the rescue management for pneumothorax after retrolaminar block. Sri Lankan $J$ Anaesthesiol. 2020; 28(2):143-145. https://doi.org/10.4038/slja.v28i2.8516

2. Damjanovska M, Stopar Pintaric T, Cvetko E, Vlassakov K. The ultrasound-guided retrolaminar block: volume-dependent injectate distribution. J Pain Res. 2018; 11:293-9.

https://doi.org/10.2147/JPR.S153660

PMID: 29445296

3. $\mathrm{Wu} \mathrm{Q}$, Zhang $\mathrm{H}$. Carbon dioxide pneumothorax following retroperitoneal laparoscopic partial nephrectomy: a case report and literature review. $B M C$ Anesthesiol. 2018; 18(1):202. https://doi.org/10.1186/s12871-018-0662-x PMID: 30579345

4. Kessler J, Schafhalter-Zoppoth I, Gray AT. An ultrasound study of the phrenic nerve in the posterior cervical triangle: implications for the interscalene brachial plexus block. Reg Anesth Pain Med. 2008; 33(6):545-550. https://doi.org/10.1136/rapm-00115550200811000-00006 PMID: 19258969.

\title{
The Authors' Reply to Comments Made on the Article "Phrenic Nerve Block, the Rescue Management for Pneumothorax After Retrolaminar Block"
}

\author{
Priyanka Mishra ${ }^{I^{*}}$, Praveen Talwar ${ }^{2}$, Nishith Govil ${ }^{3}$, Prabakaran Parameswaran ${ }^{4}$ \\ Senior Resident ${ }^{l}$, Associate Professor, ${ }^{2,3}$, Junior Resident ${ }^{4}$ All India Institute of Medical Sciences, \\ Uttarakhand, India
}

\section{Reply for comment number 1}

We acknowledge the fact that in the current scenario, multiple causes could have led to pneumothorax. However, the presence of bulla was not seen in pre-operative Chest X-ray (rules out large bulla). CT- thorax could have been beneficial in such case.
However, due to no indication, CT-thorax was not done in this patient. Moreover, though a rare possibility, the diagnosis was made as per the most probable diagnosis.

\section{Reply for comment number 2}

We understand that USG-guided RLB has 
better safety and efficacy. However, the USG machine was not accessible at that time within OT complex.

\section{Reply for comment number 3}

The presence of pneumoperitoneum during robotic laparoscopic surgery is associated with changes in respiratory mechanics. The rise in airway pressures and end-tidal $\mathrm{CO}_{2}$ during pneumoperitoneum, increases further with Trendelenburg position. ${ }^{1}$ Hence, we did not suspect pneumothorax intra-operatively as these changes were already expected. There were no significant haemodynamic changes intraoperatively.

\section{Reply for comment number 4}

Phrenic nerve block (PNB) causes an ipsilateral hemi-diaphragmatic palsy. This is the mechanism which promotes symphysis of two pleura due to decreased chest volume. Moreover, as the pre-operative lung function status was normal, unilateral diaphragmatic palsy was not considered detrimental for the patient.

\section{Reply for comment number 6}

This is one different scenario and explanation given in presence of tension pneumothorax. However, as there were no features of hypotension or pleuritic chest pain, tension pneumothorax was ruled out.

\section{Reply for comment number 7}

The PNB in this context contributes to pleural leak healing with added benefit of relieving pain. $\mathrm{We}$ have already acknowledged in our article that the therapeutic use of PNB in different types of pneumothorax needs to be further researched in bigger sample sizes, so as to establish its significance.

\section{References}

1.Brandão JC, Lessa MA, Motta-Ribeiro G, et al. Global and Regional Respiratory Mechanics During Robotic-Assisted Laparoscopic Surgery: A Randomized Study. Anesth Analg. 2019 Dec; 129(6): 1564-1573.

https://doi.org/10.1213/ANE.0000000000 004289 Erratum in: Anesth Analg. 2020 Apr; 130(4):e118. PMID: 31743177 